\title{
A Versatile and Scalable Strategy to Discrete Oligomers
}

Jimmy Lawrence ${ }^{\dagger}$, Sang-Ho Lee ${ }^{\dagger}$, Allison Abdilla ${ }^{\dagger}$, Mitchell D. Nothling ${ }^{\dagger \dagger}$, Jing M. Ren ${ }^{\dagger}$, Abigail S. Knight ${ }^{\dagger}$, Carolin Fleischmann ${ }^{\dagger}$, Youli $\mathrm{Li}^{\dagger}$, Austin S. Abrams ${ }^{\dagger}$, Bernhard V. K. J. Schmidt $^{+\#}$, Michael C. Hawker ${ }^{\dagger}$, Luke A. Connal ${ }^{\ddagger}$, Alaina J. McGrath ${ }^{\dagger}$, Paul G. Clark ${ }^{\S}$, Will R. Gutekunst ${ }^{\dagger^{*}}$, Craig J. Hawker ${ }^{\dagger^{*}}$

${ }^{\dagger}$ Materials Research Laboratory and Departments of Materials, Chemistry and Biochemistry, University of California, Santa Barbara, California 93106, United States, ${ }^{\ddagger}$ Department of Chemical Engineering, University of Melbourne, Victoria, Australia, "Max Planck Institute of Colloids and Interfaces, 14424 Potsdam, Germany, ${ }^{\S}$ The Dow Chemical Company, Midland, Michigan 48674, United States

\section{Supporting Information}

\section{TABLE OF CONTENTS}

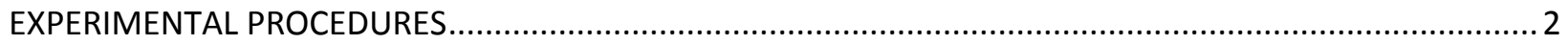

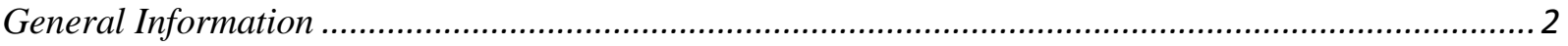

Oligomer separation: parameters for automated gradient chromatography................................. 5

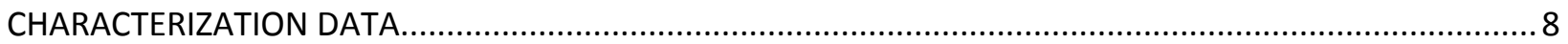

TLC and SEC analysis of oligoTBA after low resolution separation ( 12 fractions) ........................8

${ }^{1} \mathrm{H}$ NMR analysis of discrete oligoTBA after high resolution separation ( 8o fractions) .................. 9

XRD analysis of spherulitic, discrete oligoTBA samples ........................................................... 9

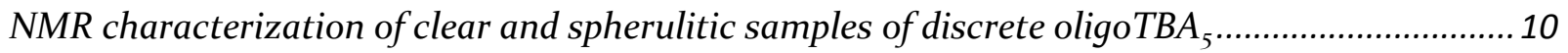

Glass transition temperature of discrete oligoTBA species $(n=3$ to 10$)$....................................... 11

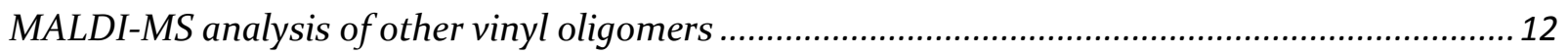

Verification of mass spectrometry techniques to analyze oligoTBA fractions................................13 


\section{EXPERIMENTAL PROCEDURES}

\section{General Information}

All reactions were carried out under an inert atmosphere of dry argon in flame-dried glassware, unless the reaction procedure states otherwise. Room temperature reactions were carried out between $21-24{ }^{\circ} \mathrm{C}$. All reagents were purchased from Sigma-Aldrich and used without further purification, unless stated otherwise. Prior to polymerization, all monomers ( $t$-butyl acrylate, styrene, 4-acetoxystyrene, methyl methacrylate) were passed through a plug of basic/neutral alumina to remove inhibitors. Anhydrous solvents were purchased from Sigma-Aldrich or taken from a solvent purification system. Copper(I) bromide $(\mathrm{CuBr})$ was purified by successive washing with glacial acetic acid, ethanol, ether, followed by drying and storage under Ar. 2,2'-Azobisisobutyronitrile (AIBN) was purified by recrystallization in $\mathrm{MeOH}$.

All chromatographic solvents were of ACS grade or better and used without further purification. Automatic flash chromatography of the oligomers was performed on Biotage $\mathrm{SP}_{1}$, Biotage $\mathrm{SP}_{4}$ or Biotage Isolera unit, using Biotage KP-SIL SNAP/SNAP Ultra cartridge series ( $25 \mathrm{~g} / 50 \mathrm{~g} / 340 \mathrm{~g}$ ) or SNAP Ultra C18 (6o g/40o g), eluting with suitable solvent pairs (Table $\mathrm{S}_{3}, \mathrm{~S}_{5}-\mathrm{S} 8$ ).

Analytical thin-layer chromatography (TLC) was performed using commercial TLC plates (EMD Millipore) and visualized using combinations of UV, bromocresol green, potassium permanganate, ceric ammonium molybdate and iodine staining. ${ }^{1} \mathrm{H}$ and ${ }^{13} \mathrm{C}$ NMR spectra were recorded on a Varian 40o, 50o, and $600 \mathrm{MHz}$ spectrometer, using the solvent signal for calibration. IR spectra were obtained using a Perkins Elmer Spectrum Fourier-Transform Infrared (FTIR) spectrometer. Gel permeation chromatography (GPC) was performed on a Waters 2695 separation module with a Waters 2414 refractive index detector eluting with $0.25 \mathrm{wt} \%$ triethylamine/chloroform. Weight average molecular weights $\left(M_{w}\right)$ and number average molecular weights $\left(M_{n}\right)$ were calculated relative to linear polystyrene standards. Reported molecular weights $\left(M_{n}\right)$ were also calculated using ${ }^{1} \mathrm{H}$ NMR by comparing the integration of the protons on the initiating chain end to the polymer pendent groups unless otherwise noted, and by mass spectral analysis. Mass spectral data were obtained at the Mass Spectrometry Facility at the University of California, 
Santa Barbara and University of California, Irvine. MALDI-TOF-MS spectra of oligo(TBA) and oligo(AcSty) were taken using 2,5-dihydroxybenzoic acid (DHB) /sodium trifluoroacetate (Na-TFA) matrix. MALDITOF-MS spectra of oligo(Sty) and oligo(MMA) were taken using dithranol/Na-TFA.

\section{Oligomer synthesis (CRP)}

A $40 \mathrm{~mL}$ reaction vial equipped with a stir bar and a septa cap (100 $\mathrm{mL}$ Schlenk flask for large scale reactions) was flame dried and subjected to vacuum-Ar filling cycles three times. Inhibitor-free monomer, $N, N, N^{\prime}, N^{\prime \prime}, N^{\prime \prime}$-pentamethyldiethylenetriamine, $\mathrm{CuBr}$ with a 9:0.5:0.5 ratio were added to the vial and stirred.

The mixture was cooled in an ice bath and degassed by $\mathrm{Ar}$ bubbling for 20 minutes. For the oligomerizations of t-butyl acrylate, acetone ( 18 vol\%) was added as co-solvent. After degassing, the reaction mixture was then added with 1 equiv. of degassed ATRP initiator (ethyl $\alpha$-bromoisobutyrate (EBiB)) or RAFT-chain transfer agent (2-(dodecylthiocarbonothioylthio)-2-methyl propionic acid) to start the polymerization. The polymerization was terminated by thermal quenching in liquid nitrogen and opening the reaction vial to ambient atmosphere. The resulting viscous mixture was diluted with DCM, passed through a basic alumina plug and reduced in vacuo to obtain the crude oligomers.

Table S1. Oligomerization conditions for vinyl monomers (ATRP)

\begin{tabular}{ccc}
\hline Monomer & Time $(\mathrm{h})$ & $\mathrm{T}\left({ }^{\circ} \mathrm{C}\right)$ \\
\hline$t$-Butyl acrylate (ATRP) & 0.5 & 60 \\
$t$-Butyl acrylate (RAFT) & 12 & 60 \\
Styrene & 10 & 90 \\
4-Acetoxystyrene & 10 & 90 \\
\hline
\end{tabular}




\section{Isotactic oligo(methyl methacrylate) synthesis (anionic)}

it-OMMA was synthesized by anionic polymerization according to previously published procedures with modifications. ${ }^{1.2}$ The Grignard reagent, $t-\mathrm{C}_{4} \mathrm{H}_{9} \mathrm{MgBr}$, was prepared as follows: anhydrous DEE $(28 \mathrm{~mL})$ and $t$ butylbromide ( $14 \mathrm{~g}, 0.10 \mathrm{~mol}$ ) were added consecutively into a dry addition funnel under $\mathrm{N}_{2}$. Separately, $\mathrm{Mg}$ turnings ( $3.73 \mathrm{~g}, 0.15 \mathrm{~mol}$ ) were added to a dry round bottom flask under $\mathrm{N}_{2}$ via a funnel with a flushing adapter, followed by the addition of anhydrous DEE ( $60 \mathrm{~mL})$. The $t$-butylbromide solution was then added slowly at $25{ }^{\circ} \mathrm{C}$ over $1 \mathrm{~h}$. The solution was stirred for a further hour and then left to stand for 12 hours. The Grignard reagent $\left(\left[t-\mathrm{C}_{4} \mathrm{H}_{9} \mathrm{MgBr}\right]_{e f f}=358 \mathrm{mM}\right.$ determined via polymerization $)$ was stored in a dry roundbottom flask equipped with a 3-way stopcock under Ar atmosphere at o ${ }^{\circ} \mathrm{C}$ before use.

The general procedure employed for the synthesis of it-OMMA: the initiator solution (10 $\mathrm{mL}$ ) was added to anhydrous toluene $(57 \mathrm{~mL})$ in a Schlenk tube at $-78^{\circ} \mathrm{C}$ under Ar. MMA was then added dropwise ( $4.6 \mathrm{~mL}$ in $5 \mathrm{~min}$ ) causing the mixture to turn orange. The polymerization solution was kept at $-78{ }^{\circ} \mathrm{C}$ for $30 \mathrm{~h}$, and then degassed anhydrous methanol $(5 \mathrm{~mL})$ was added to the Schlenk flask to quench the reaction. The reaction solution was diluted with toluene $(50 \mathrm{~mL})$, washed with $2 \mathrm{M} \mathrm{HCl}(50 \mathrm{~mL} \times 3)$ and distilled water $(50 \mathrm{~mL} \times 3)$, dried $\left(\mathrm{MgSO}_{4}\right)$, filtered and concentrated to obtain it-OMMA as a waxy solid, $3.50 \mathrm{~g}(83 \%) ;{ }^{13} \mathrm{C}$ NMR: $m m / m r / r r(\%)=80 / 10 / 10$.

Table S2. Size-exclusion chromatography analysis of the vinyl oligomers

\begin{tabular}{lcc}
\hline \multicolumn{1}{c}{ Oligomer } & $M_{n \text { SEC, } \mathrm{CHCl}_{3}}$ & $Ð$ \\
\hline Oligo(t-butyl acrylate) (ATRP) & $1.4 \mathrm{kDa}$ & 1.2 \\
Oligo(t-butyl acrylate) (RAFT) & $0.8 \mathrm{kDa}$ & 1.2 \\
Oligo(methyl methacrylate) (anionic) & $2.7 \mathrm{kDa}$ & 1.2 \\
Oligo(styrene) (ATRP) & $1.1 \mathrm{kDa}$ & 1.2 \\
Oligo(4-acetoxystyrene) (ATRP) & $1.9 \mathrm{kDa}$ & 1.2 \\
\hline
\end{tabular}




\section{Oligomer separation: parameters for automated gradient chromatography}

A Biotage SP1 flash purification system was equipped with an appropriate column cartridge (KP SIL or SNAP Ultra, size $=25 \mathrm{~g} / 50 \mathrm{~g} / 340 \mathrm{~g})$ or SNAP Ultra C18 $(60 \mathrm{~g} / 400 \mathrm{~g})$. The column was equilibrated with 3 column volumes of the weak solvent. (for KP SIL-25 g, $1 \mathrm{CV}=33 \mathrm{~mL}$ ). After equilibration is complete, the screw-top cap of the column and the solvent dispersant head insert were detached and set aside (Figure $\mathbf{S} \mathbf{1}$ a-c). The small volume of remaining solvent was removed, and the crude oligomer sample dissolved in the weak solvent (e.g., hexane, toluene, $100 \mathrm{mg} / \mathrm{mL}$ ) was added carefully onto the frit inside the column (Figure S1 d, e). For cartridges other than $340 \mathrm{~g}$, the sample was loaded into the column by pressing the "Load Sample" command. For 340 g cartridge, the sample was loaded by bleeding the solvent from the bottom of the column. After the sample was successfully loaded onto the column (Figure $\mathbf{S} \mathbf{f}, \mathbf{g}$ ), a few $\mathrm{mL}$ of weak solvent were added to wash the cartridge and complete the loading process (Figure $\mathbf{S} \mathbf{1} \mathbf{h}$ ). The

upper assembly (screw-top cap and solvent dispersant head) was re-attached (Figure Si i), “Close” and “Gradient" buttons were pressed to start the elution following a programmed gradient (Table $\mathbf{S}_{\mathbf{3}}$, $\mathbf{S}_{\mathbf{5}}$-S8). The flow rate and fraction size were set according to the cartridge size (Table $\mathbf{S}_{\mathbf{4}}$ ). 

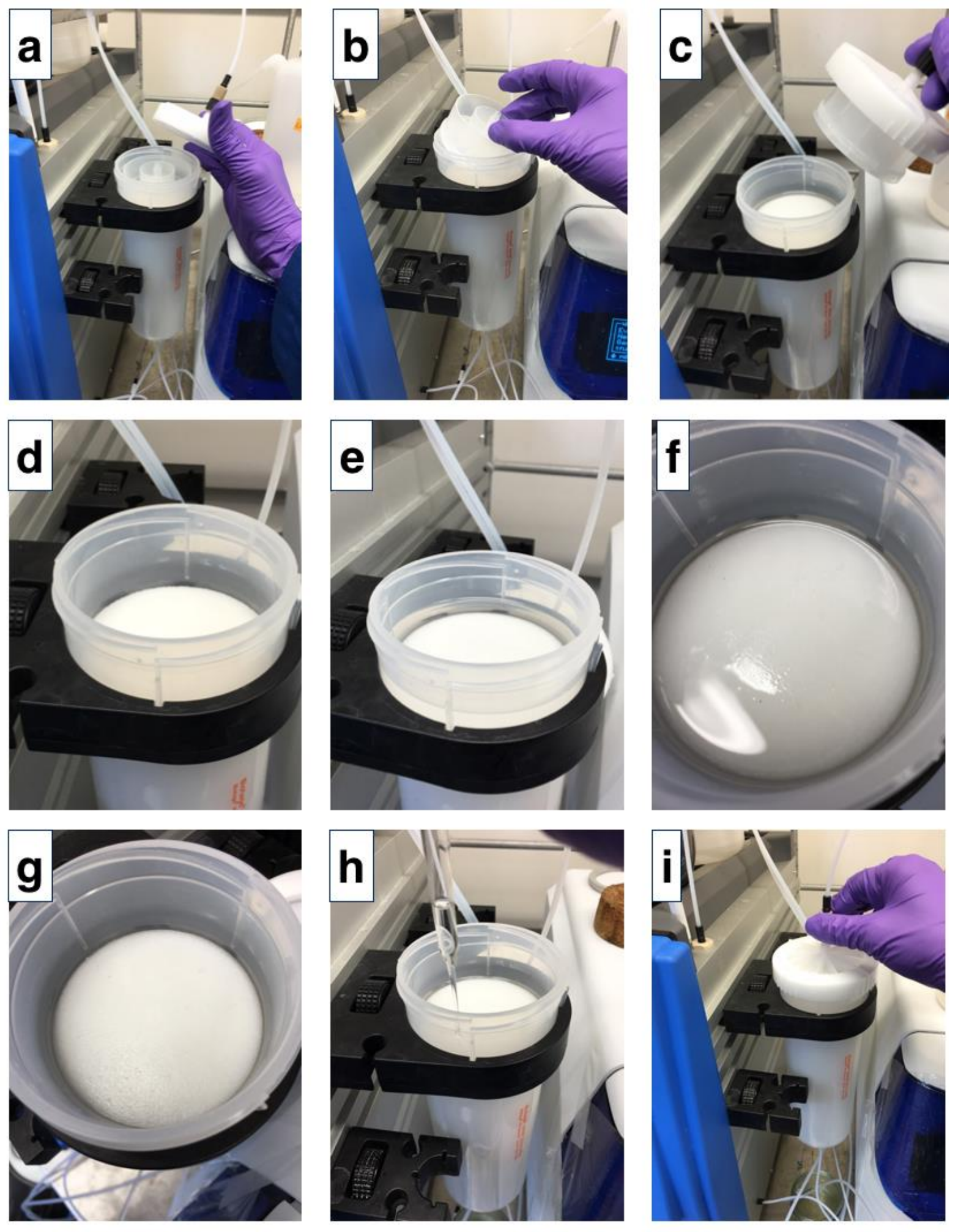

Figure S1. (a-i) Procedures for loading the crude oligomer sample onto the column. 
Table S3. Gradient profile for oligoTBA separation (eluent: hexane/ethyl acetate)

\begin{tabular}{ccc}
\hline \%EtOAc (start) & \%EtOAc (end) & Column Volume (CV) \\
\hline o & 5 & 2 \\
5 & 25 & 12 \\
25 & 100 & 4 \\
\hline
\end{tabular}

Table S4. Flow rate and fraction size for oligomer separation $(\mathrm{RP}=$ reversed phase $)$

\begin{tabular}{cccc}
\hline Column & Loading $(\mathrm{g})$ & Flow rate $(\mathrm{mL} / \mathrm{min})$ & Fraction size $(\mathrm{mL})$ \\
\hline KP-SIL 25 g & 0.5 & 25 & $15-20$ \\
KP-SIL 50 g & 1.0 & 40 & 20 \\
KP-SIL 340 g & 7.0 & 80 & 50 \\
SNAP Ultra 340 g & 15 & 200 & 50 \\
SNAP Ultra C18 6o g (RP) & 2.0 & 40 & 20 \\
SNAP Ultra C18 400 g (RP) & 12.0 & 200 & 50 \\
\hline
\end{tabular}

Table S5. Gradient profile for oligo(styrene) separation (reverse phase, eluent: $\mathrm{MeCN} / \mathrm{Hex}$ )

\begin{tabular}{ccc}
\hline \%THF (start) & \%Hex (end) & Column Volume $(\mathrm{CV})$ \\
\hline 0 & 0 & 6 \\
0 & 20 & 12 \\
20 & 26 & 3 \\
\hline
\end{tabular}

Table S6. Gradient profile for oligo(4-acetoxystyrene) separation (eluent: toluene/THF)

\begin{tabular}{ccc}
\hline \%THF (start) & \%THF (end) & Column Volume (CV) \\
\hline o & 2 & 2 \\
2 & 15 & 13 \\
15 & 15 & 5 \\
\hline
\end{tabular}

Table S7. Gradient profile for oligo(methyl methacrylate) separation (eluent: toluene/MeCN)

\begin{tabular}{ccc}
\hline$\% \mathrm{MeCN}$ (start) & \%MeCN (end) & Column Volume (CV) \\
\hline $\mathrm{o}$ & 25 & 15 \\
25 & 50 & 10 \\
\hline
\end{tabular}


Table S8. Gradient profile for PDMS-OH separation (eluent: MeOH/hexane)

\begin{tabular}{ccc}
\hline \%Hex (start) & \%Hex (end) & Column Volume $(\mathrm{CV})$ \\
\hline $\mathrm{o}$ & 10 & 10 \\
10 & 10 & 10 \\
\hline
\end{tabular}

\section{CHARACTERIZATION DATA}

TLC and SEC analysis of oligoTBA after low resolution separation ( 12 fractions)

$T L C$

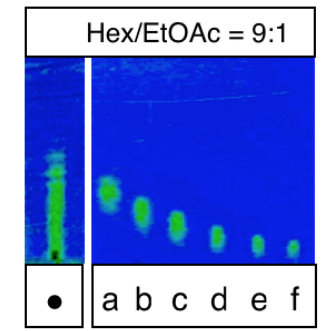

SEC

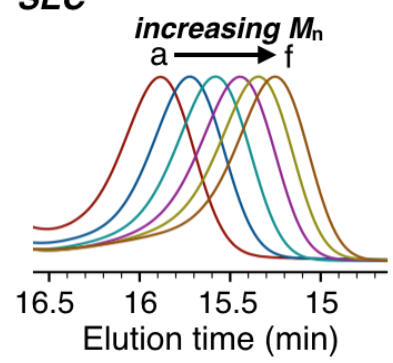

$\overline{D P}_{\text {NMR }}$

- a b c c d e $f$

$\begin{array}{llllllll}8 & 5.0 & 6.6 & 8.3 & 9.5 & 11 & 12\end{array}$
MALDI-TOF-MS

a
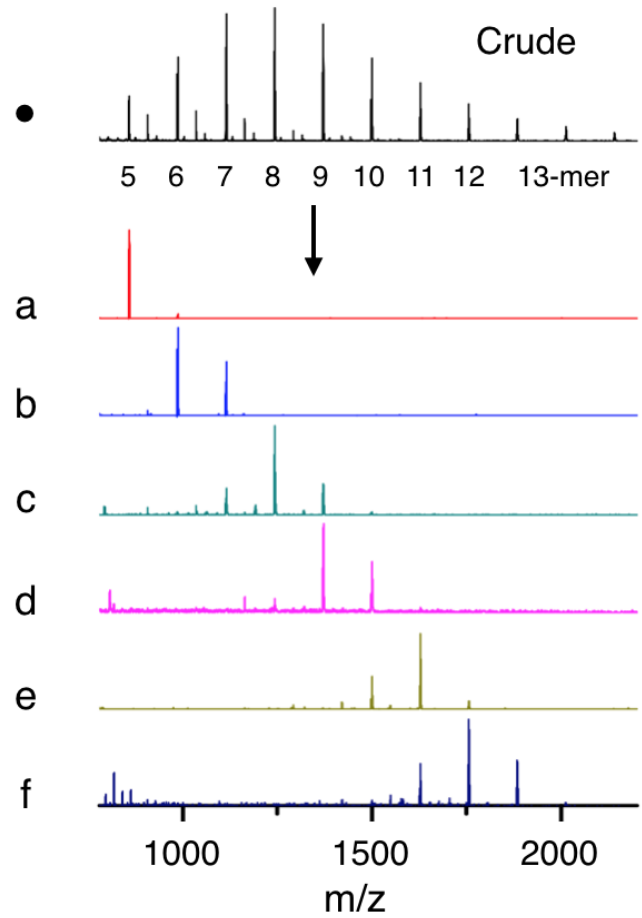

Figure S2. TLC, SEC, ${ }^{1} \mathrm{H}$ NMR and MALDI-TOF-MS analysis of crude oligoTBA $(\bullet)$ and oligoTBA fractions (a-f) after low resolution separation ( $\sim 12$ fractions) 
${ }^{1} \mathrm{H}$ NMR analysis of discrete oligoTBA after high resolution separation ( $\sim 80$ fractions)
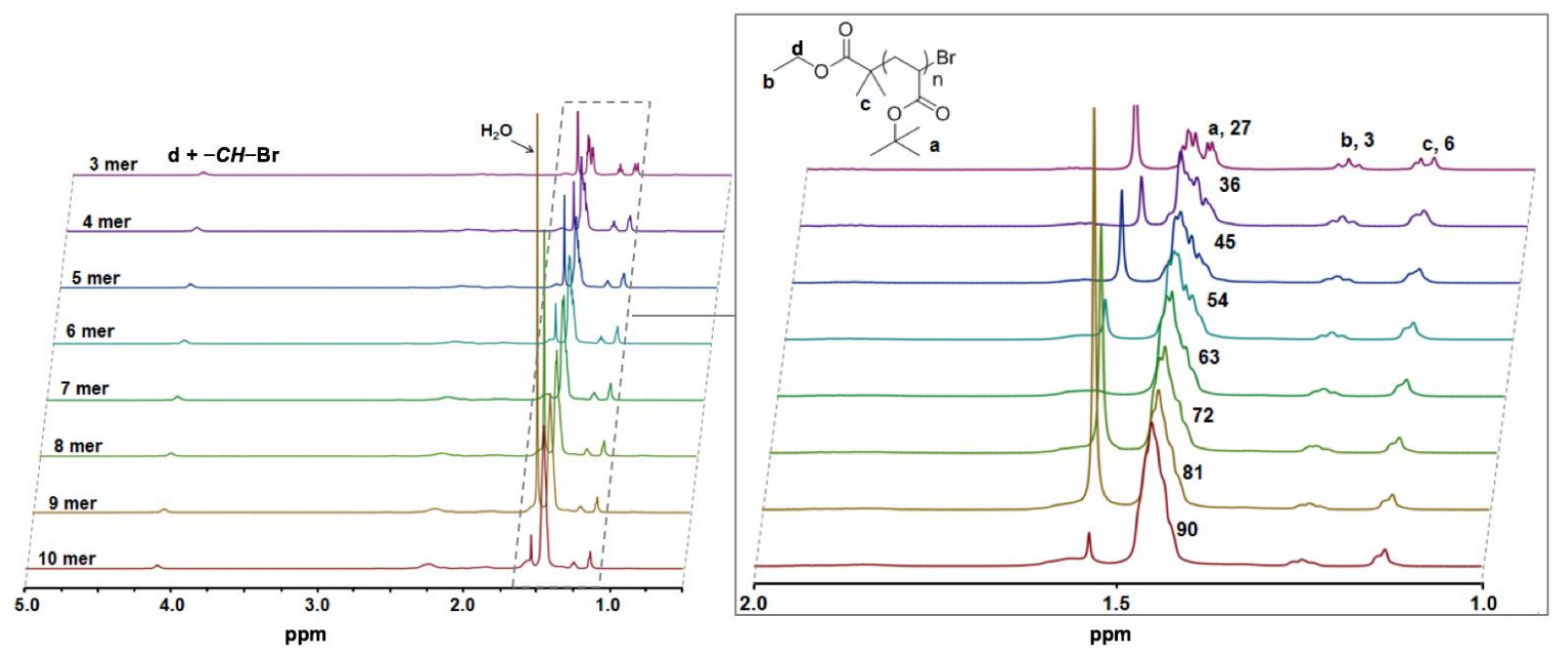

Figure S3. ${ }^{1} \mathrm{H}$ NMR spectra of discrete oligoTBA (trimer to decamer). Inset shows the area corresponding to t-butyl protons at $1.45 \mathrm{ppm}$ ('a'). Integration of the $t$-butyl signal is shown as $(9 \times \mathrm{n})$, normalized to the initiator protons ('b' and 'c').

XRD analysis of spherulitic, discrete oligoTBA samples

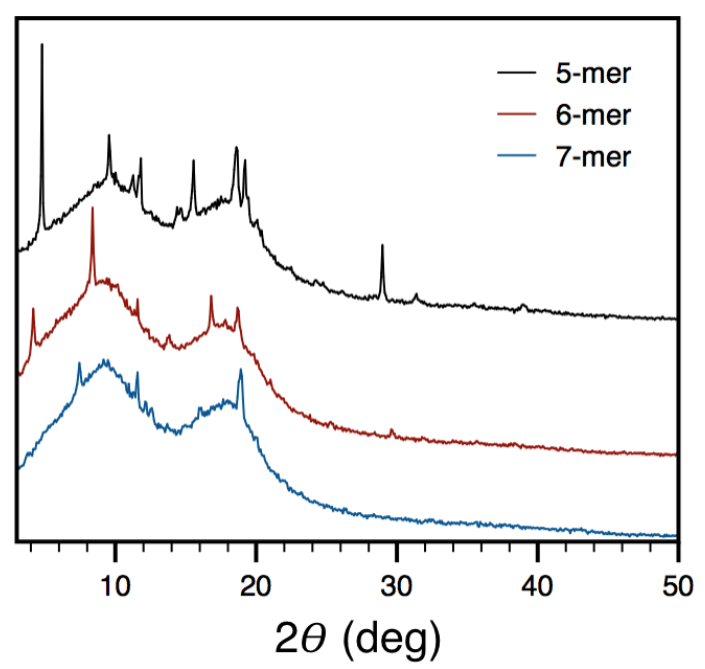

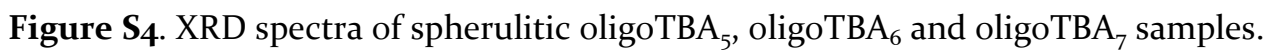


NMR characterization of clear and spherulitic samples of discrete oligoTBA $A_{5}$

(a)
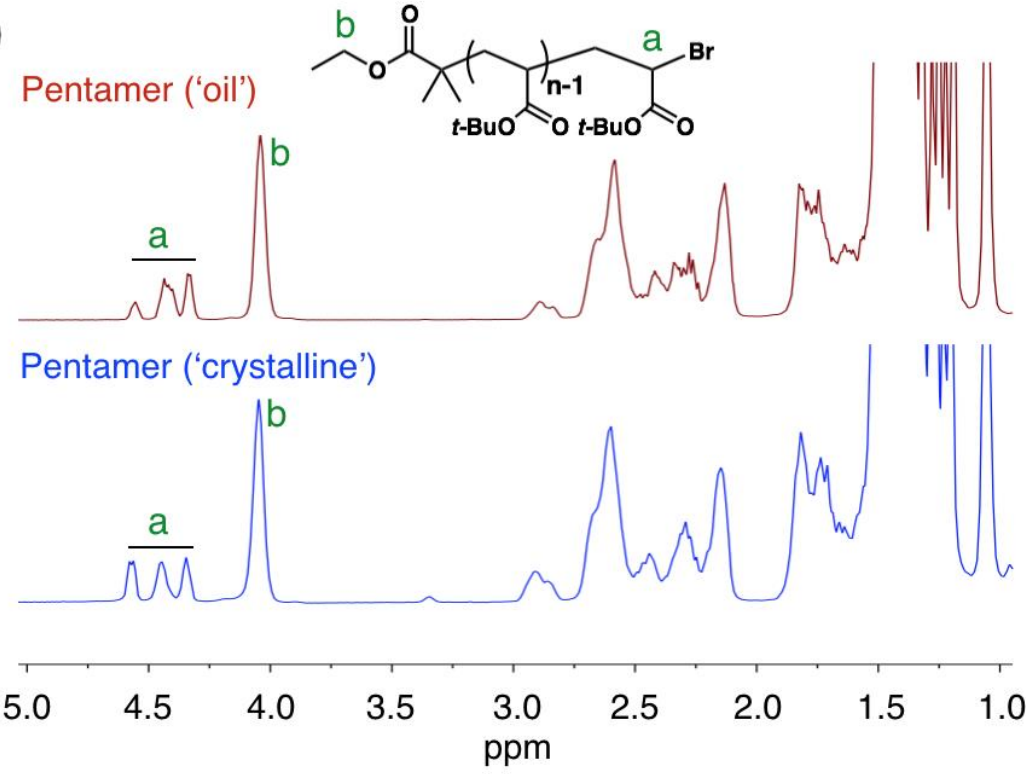

(b)

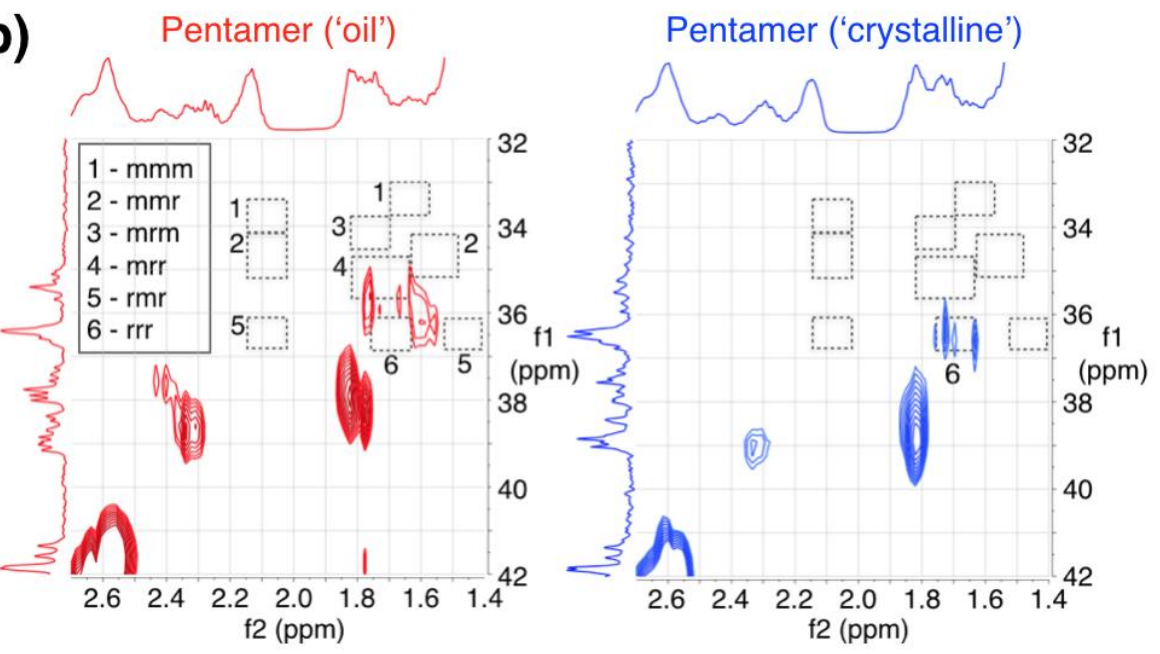

Figure S5. (a) ${ }^{1} \mathrm{H}$ NMR and (b) $2 \mathrm{D}$ HMQC-NMR analysis of oil-like and spherulitic samples of oligoTBA $\left(\mathrm{C}_{6} \mathrm{D}_{6}, 1 \mathrm{~mol} \% \mathrm{Cr}(\mathrm{acac})_{3}\right)$. Tetrads were assigned according to the literature (Macromolecules 1993, 26, 102106). 
Glass transition temperature of discrete oligoTBA species $(n=3$ to 1o)

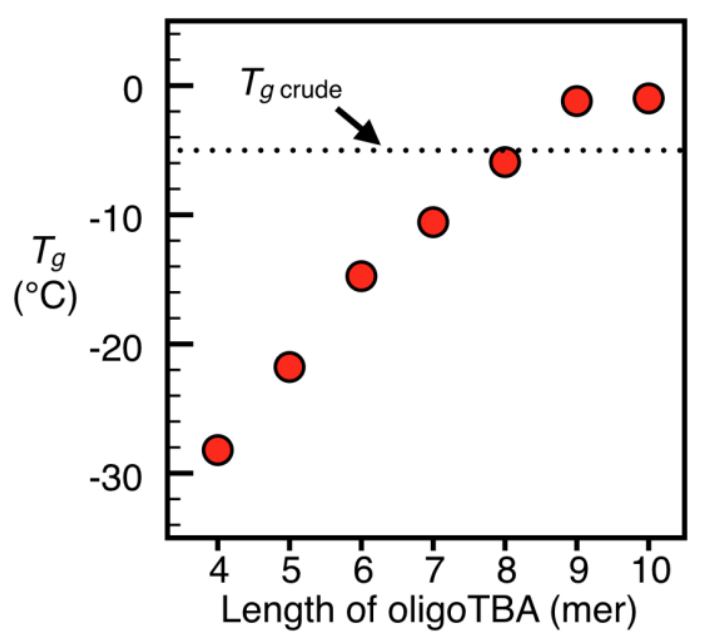

Figure S6. Glass transition temperature $\left(T_{g}\right)$ of discrete oligoTBA 4 (tetramer) to oligoTBA ${ }_{10}$ (decamer). 
MALDI-MS analysis of other vinyl oligomers

(a)

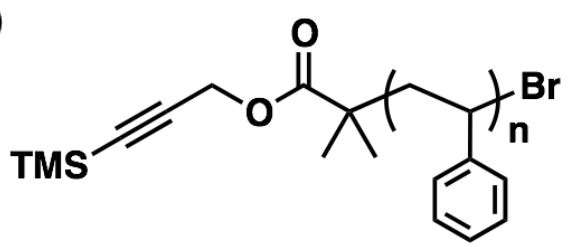

TMS-alkyne oligoSty

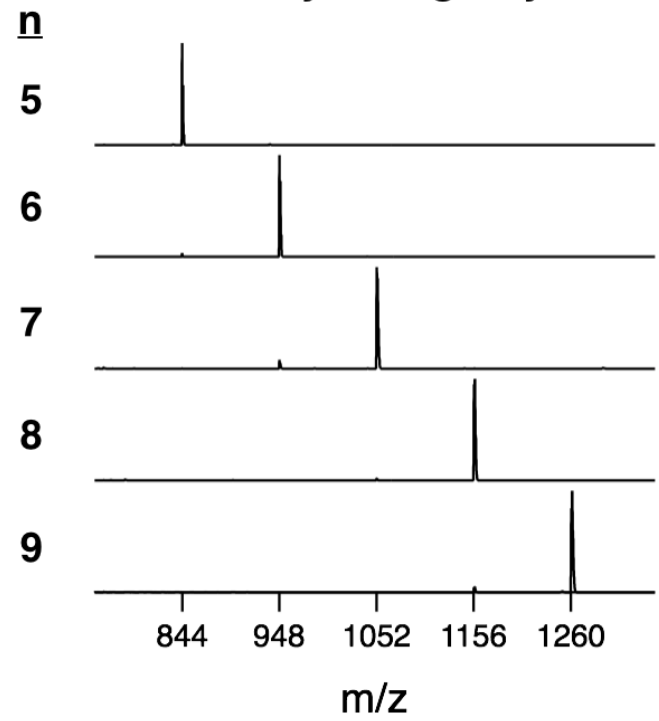

(b)<smiles>COC(=O)C1=CCC1C=CC(C)(C)C</smiles>

it-oligoMMA

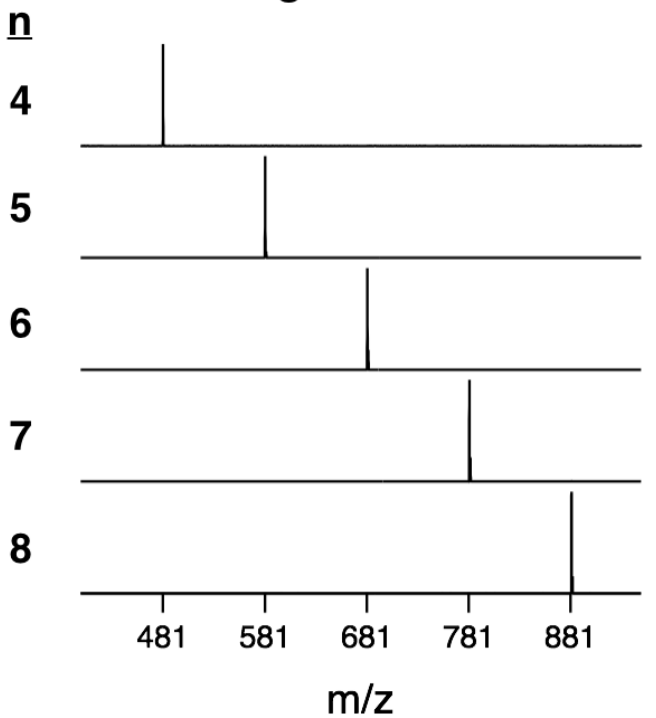

Figure S7. Representative MALDI-MS spectra of styrenic and methacrylic oligomers. (a) TMS-alkyne functionalized oligo(styrene) prepared using ATRP and (b) isotactic oligo(methylmethacrylate) prepared using anionic polymerization. 
Verification of mass spectrometry techniques to analyze oligoTBA fractions

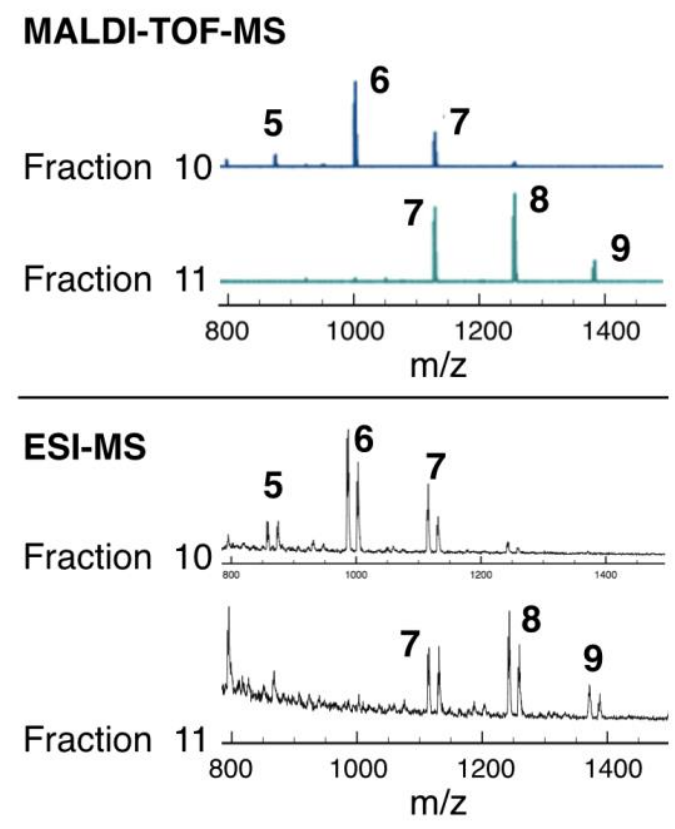

Figure S8. Verification of MS techniques to analyze oligoTBA fractions. Top: MALDI-MS, bottom: ESI-MS. The doubling of peaks in the ESI-MS spectrum is due to sodium and potassium adduct ions being present.

\section{References}

1. K. Hatada, K. Ute, K. Tanaka, T. Kitayama, Y. Okamoto, Polym. J. 1985, 17, 977-980.

2. J. M. Ren, K. Satoh, T. K. Goh, A. Blencowe, K. Nagai, K. Ishitake, A. J. Christofferson, G. Yiapanis, I. Yarovsky, M. Kamigaito and G. G. Qiao, Angew. Chem. Int. Ed., 2014, 53, 459-464. 